\title{
IR Study of Monomer-Dimer Self-association of 2,2-Dimethyl-3-ethyl-3-pentanol in $n$-Octane: Determination of the Molar Absorptivities of Monomer and Dimer Bands, and Dimerization Constants Using Novel Equations
}

\author{
Jenn-Shing Chen $\cdot$ Kwei-Tin Yeh $\cdot$ Cheng-Chang Wu \\ Dah-Yu Kao
}

Received: 12 May 2012/ Accepted: 8 January 2013/Published online: 19 November 2013

(C) Springer Science+Business Media New York 2013

\begin{abstract}
The monomer-dimer self-association equilibrium of 2,2-dimethyl-3-ethyl-3pentanol in $n$-octane has been studied by IR spectroscopy at four temperatures $(288,298$, 308 , and $318 \mathrm{~K}$ ). The solute was chosen to restrict the self-association between solute molecules to dimerization only, owing to steric hindrance of the bulky chains in the vicinity of the $\mathrm{OH}$ group. Two linear equations have been derived for the treatment of the experimental data. One of these equations was used to treat the data of the concentration dependent integrated absorbance of the monomer bands for each temperature to obtain the monomer molar absorptivity, $\varepsilon_{\mathrm{m}}$, and dimerization constant, $K$. The other equation was used to treat the data of concentration-dependent dimer bands to obtain the dimer molar absorptivity, $\varepsilon_{\mathrm{d}}$, and $K$. Thus, the dimerization constant was determined by two methods. Since the same thermodynamic quantity $K$ is obtained from either the monomer bands or the dimer bands, the difference between them at a given temperature can serve as an assessment of the quality of the experiment. The standard enthalpy and entropy of dimerization were also obtained from a van't Hoff plot.
\end{abstract}

Keywords Monomer-dimer self-association · IR spectroscopy $\cdot$ Hindered alcohols · Enthalpy and entropy of dimerization

\section{Introduction}

Hydrogen bonding is ubiquitous in the field of biological chemistry. It plays an important role in the structure of proteins [1, 2], base-pairing in DNA [3], and enzyme kinetics. Hydrogen bonding is also considered to be one of the major factors needed to describe solutions deviating from normal behavior [4], such as abnormal elevation of the boiling point and lowering of the vapor pressure. Studies of hydrogen bonding are quite extensive

J.-S. Chen $(\bowtie) \cdot$ K.-T. Yeh $(\bowtie) \cdot$ C.-C. Wu · D.-Y. Kao

Department of Applied Chemistry, National Chiao-Tung University, Hsin-Chu 30050, Taiwan e-mail: jschen@cc.nctu.edu.tw

K.-T. Yeh

e-mail: ralf.ac98g@nctu.edu.tw 
using various experimental methods as well as theoretical calculations [5-11]. Among the experimental methods, IR spectroscopy is considered to be the most convenient and a powerful one, ever since the establishment of the IR spectral characteristics of hydrogen bonding in alcohols by Errera and Mollet [12]. However, in addition to the band attributed to non hydrogen-bonded monomeric species, IR spectra may be complicated by displaying several overlapped bands corresponding to hydrogen-bonded species of various sizes and configurations due to dimerization or oligomerization. In this case, the study of selfassociation by IR is quite difficult in that a conjecture is needed as to what sizes and what configurations occur in the system, in order to interpret the spectra [13-19]. As a rule, conjectures are prone to inaccuracies, leading to an unreliable subsequent determination of spectral and thermodynamic parameters. Nevertheless, accurate determination of spectral and thermodynamic parameters can be still made for systems exhibiting only monomerdimer self-association. This can be realized using hindered alcohols in dilute solution, with bulky side chains in the vicinity of the $\mathrm{OH}$ group. The steric hindrance due to the bulky side chains hampers alcohol-alcohol oligomerization [19-23].

In this report we choose dilute solutions of 2,2-dimethyl-3-ethyl-3-pentanol in $n$-octane as a model system to study monomer-dimer self-association [23-26]. In this system, no oligomer bands appear and monomer bands and dimer bands exhibit little overlap, which makes them amenable to spectral deconvolution. To achieve a better determination, we have derived two linear utility equations, one for the data treatment of the monomer bands (Eq. 9 below) to obtain the molar absorptivity of the monomer band, $\varepsilon_{\mathrm{m}}$, and the dimerization constant, $K$, for each temperature; the other equation for dimer bands (Eq. 13 below) was used to obtain the molar absorptivity of the dimer band, $\varepsilon_{\mathrm{d}}$, and the dimerization constant, $K$, for each temperature. It should be remarked that the same dimerization constant can be determined either from the monomer bands or the dimer bands.

\section{Utility Equations for the Data Treatment}

In a monomer-dimer self-association equilibrium, the monomer, $\mathrm{B}$, and the dimer, $\mathrm{B}_{2}$, exhibit a dynamic equilibrium:

$$
\mathrm{B}+\mathrm{B} \rightleftharpoons \mathrm{B}_{2}
$$

The concentration of the monomer, $[\mathrm{B}]$, and that of the dimer, $\left[\mathrm{B}_{2}\right]$, are related by

$$
[\mathrm{B}]+2\left[\mathrm{~B}_{2}\right]=[\mathrm{B}]_{\mathrm{o}}
$$

where $[\mathrm{B}]_{\mathrm{o}}$ is the initially (prepared) concentration. In addition to this restriction, the equilibrium concentrations of $[\mathrm{B}]$ and $\left[\mathrm{B}_{2}\right]$ are related by a temperature-dependent dimerization constant,

$$
\mathrm{K}=\frac{\left[\mathrm{B}_{2}\right]}{[\mathrm{B}]^{2}}
$$

Combining Eqs. 2 and 3 permits us to solve for $[\mathrm{B}]$ and $\left[\mathrm{B}_{2}\right]$ in terms of $K$ and $[\mathrm{B}]_{\mathrm{o}}$ :

$$
\begin{gathered}
{[\mathrm{B}]=\frac{2[\mathrm{~B}]_{\mathrm{o}}}{\left(1+8 K[\mathrm{~B}]_{\mathrm{o}}\right)^{1 / 2}+1}} \\
{\left[\mathrm{~B}_{2}\right]=\left(\frac{1}{2}\right) \frac{\left(1+8 K[\mathrm{~B}]_{\mathrm{o}}\right)^{1 / 2}-1}{\left(1+8 K[\mathrm{~B}]_{\mathrm{o}}\right)^{1 / 2}+1}[\mathrm{~B}]_{\mathrm{o}}}
\end{gathered}
$$


Equations 4 and 5 have also been derived elsewhere for the NMR study of monomerdimer self-association [26-29].

The absorbance for monomer, $A_{\mathrm{m}}(\tilde{v})$, at a particular wavelength $\tilde{v}$, according to the Beer-Lambert's law, is given by:

$$
A_{\mathrm{m}}(\tilde{v})=\varepsilon_{\mathrm{m}}(\tilde{v}) b[\mathrm{~B}]
$$

where $\varepsilon_{\mathrm{m}}(\tilde{v})$ is the molar absorptivity of the monomer at $\tilde{v}$, and $b$ is the optical path length of the cell. The integrated absorbance of this monomer band, $A_{\mathrm{m}}$, is then obtained by integrating over the whole range of wavenumbers covered by this band, i.e.:

$$
A_{\mathrm{m}}=\int \varepsilon_{\mathrm{m}}(\tilde{v}) b[\mathrm{~B}] d \tilde{v}=\left(\int \varepsilon_{\mathrm{m}}(\tilde{v}) d \tilde{v}\right) b[\mathrm{~B}]=\frac{2 \varepsilon_{\mathrm{m}} b[\mathrm{~B}]_{\mathrm{o}}}{\left(1+8 K[\mathrm{~B}]_{\mathrm{o}}\right)^{1 / 2}+1}
$$

where Eq. 4 has been used, and $\int \varepsilon_{\mathrm{m}}(\tilde{v}) d \tilde{v}$ is denoted for brevity by $\varepsilon_{\mathrm{m}}$, the molar absorptivity of the monomer band. Equation 7 can be rearranged to obtain:

$$
\frac{2 \varepsilon_{\mathrm{m}} b[\mathrm{~B}]_{\mathrm{o}}}{A_{\mathrm{m}}}=\left(1+8 K[\mathrm{~B}]_{\mathrm{o}}\right)^{1 / 2}+1
$$

If we subtract unity from both sides of Eq. 8, followed by taking squares, we arrive at:

$$
\frac{[\mathrm{B}]_{\mathrm{o}}}{A_{\mathrm{m}}}=\frac{1}{\varepsilon_{\mathrm{m}} b}+\left(\frac{2 K}{\left(\varepsilon_{\mathrm{m}} b\right)^{2}}\right) A_{\mathrm{m}}
$$

after some simple algebraic manipulations. Equation 9 allows us to fit the experimental data of $y=\frac{[\mathrm{B}]_{o}}{A_{\mathrm{m}}}$ versus $x=A_{\mathrm{m}}$ to a straight line whose slope $p$ and intercept $q$ give $\varepsilon_{\mathrm{m}}=\frac{1}{q b}$ and $K=\frac{p}{2 q^{2}}$.

Following the same line of reasoning as in deriving Eq. 7, from Eq. 5 the integrated absorbance of the dimer band, $A_{\mathrm{d}}$, is obtained to be:

$$
A_{\mathrm{d}}=\varepsilon_{\mathrm{d}} b\left[\mathrm{~B}_{2}\right]=\left(\frac{\varepsilon_{\mathrm{d}} b[\mathrm{~B}]_{\mathrm{o}}}{2}\right) \frac{\left(1+8 K[\mathrm{~B}]_{\mathrm{o}}\right)^{1 / 2}-1}{\left(1+8 K[\mathrm{~B}]_{\mathrm{o}}\right)^{1 / 2}+1}
$$

where $\varepsilon_{\mathrm{d}}$ is the molar absorptivity of the dimer band. Dividing the first and last terms in Eq. (10) by $\varepsilon_{\mathrm{d}} b[\mathrm{~B}]_{\mathrm{o}} / 2$, and making use of the fact that if $\frac{a}{b}=\frac{c}{d}$ then $\frac{a+b}{a-b}=\frac{c+d}{c-d}$, we obtain

$$
\frac{\varepsilon_{\mathrm{d}} b[\mathrm{~B}]_{\mathrm{o}}+2 A_{\mathrm{d}}}{\varepsilon_{\mathrm{d}} b[\mathrm{~B}]_{\mathrm{o}}-2 A_{\mathrm{d}}}=\left(1+8 K[\mathrm{~B}]_{\mathrm{o}}\right)^{1 / 2}
$$

Taking squares on both sides of Eq. 11 and making use of the fact that if $\frac{a}{b}=\frac{c}{d}$ then $\frac{a-b}{b}=\frac{c-d}{d}$, we obtain

$$
\frac{\varepsilon_{\mathrm{d}} b A_{\mathrm{d}}}{K}=\left(\varepsilon_{\mathrm{d}} b[\mathrm{~B}]_{\mathrm{o}}-2 A_{\mathrm{d}}\right)^{2}
$$

We then take square roots on both sides then divide them by $[B]_{\mathrm{o}}$ on both sides to transform Eq. 12 into a linear form which is suitable for the data treatment of the integrated absorbance of the dimer band:

$$
\frac{2 A_{\mathrm{d}}}{[\mathrm{B}]_{\mathrm{o}}}=\varepsilon_{\mathrm{d}} b-\left(\frac{\varepsilon_{\mathrm{d}} b}{K}\right)^{1 / 2} \frac{\left(A_{\mathrm{d}}\right)^{1 / 2}}{[\mathrm{~B}]_{\mathrm{o}}}
$$


The experimental data of $y=\frac{2 A_{\mathrm{d}}}{[\mathrm{B}]_{\mathrm{o}}}$ versus $x=\frac{\left(A_{\mathrm{d}}\right)^{1 / 2}}{[\mathrm{~B}]_{\mathrm{o}}}$ is then fitted to Eq. 13 to obtain the best values of $\varepsilon_{\mathrm{d}}=\frac{q}{b}$ and $K=\frac{q}{p^{2}}$ from the slope $p$ and intercept $q$ of the regressed line.

It is remarkable that the dimerization constant $K$ can be independently determined either from the data treatment of the monomer band based on Eq. 9, or from the data treatment of the dimer band based on Eq. 13.

\section{Experimental Section}

The solute 2,2-dimethyl-3-ethyl-3-pentanol ( $\geq 98 \%$ ) and the solvent $n$-octane (quoted $\geq 97 \%$ in catalogue, actual assay $98.989 \%$ by supplier) were purchased from Aldrich and Tedia, respectively, and were used without further purification. We prepared, using an analytical balance, using vials and microsyringes, the sample concentrations on the molality scale. The molalities were then converted into concentrations on the molarity scale based on the density of the solvent at different temperatures $\left(288 \mathrm{~K}, 0.707 \mathrm{~g} \cdot \mathrm{cm}^{-3}\right.$; $298 \mathrm{~K}, 0.701 \mathrm{~g} \cdot \mathrm{cm}^{-3} ; 308 \mathrm{~K}, 0.699 \mathrm{~g} \cdot \mathrm{cm}^{-3} ; 318 \mathrm{~K}, 0.683 \mathrm{~g} \cdot \mathrm{cm}^{-3}$ ), which were obtained from interpolating the published data [30, 31]. Subsequent calculations, listing and graphical plotting were then carried out in terms of molarity scale, as needed.

The spectra were taken with a FTIR spectrometer (Bio-Red Spc. 3200) with a $\mathrm{CaF}_{2}$ cell window. The cell temperature was controlled by circulating the water from a thermostat around the cell jacket, and was measured by a thermocouple inserted into the jacket of the cell. The uncertainty of the cell temperature was estimated to be $\pm 1 \mathrm{~K}$. A personal computer running the commercial software package PeakSolve (version 6.0, GRAMS, 2000, Galatic Industries Corp.) was connected to the spectrometer to perform the task of displaying, resolving the spectra, and calculating the integrated absorbance of the resolved spectra. Subsequent work, such as linear regression based on Eqs. 9 and 13 and plotting graphs, were carried out on another personal computer using the commercial software Mathematica.

\section{Results and Discussion}

Figure 1a presents a collection of the spectra exhibited by the $\mathrm{OH}$ fundamental stretching for different concentrations of 2,2-dimethyl-3-ethyl-3-pentanol in $n$-octane at $298 \mathrm{~K}$. Two bands are observed for each concentration: the sharp one at higher wavenumber, ca. $3,630 \mathrm{~cm}^{-1}$, corresponds to the $\mathrm{OH}$ fundamental stretching in the monomeric species, whereas the broad one at lower wavenumber, ca. $3,530 \mathrm{~cm}^{-1}$, corresponds to that for the dimeric species. These two bands are not well separated. They were resolved, and their respective integrated absorbance were calculated with the commercial software PeakSolve (version 6.0, GRAMS, 2000, Galatic Industries Corp.) A typical result of this resolution is presented in Fig. 1b for the concentration $0.2798 \mathrm{~mol} \cdot \mathrm{L}^{-1}$ at $298 \mathrm{~K}$. The calculated integrated absorbance for the monomer bands, $A_{\mathrm{m}}$, and those for the dimer bands, $A_{\mathrm{d}}$, are listed in Table 1 for different concentrations at the four studied temperatures, 288, 298, 308, and $318 \mathrm{~K}$.

From Table 1 it is seen that, at a given temperature, both $A_{\mathrm{m}}$ and $A_{\mathrm{d}}$ increase with the initial solute concentration, $[\mathrm{B}]_{\mathrm{o}}$. However, the observation that $A_{\mathrm{d}} / A_{\mathrm{m}}^{2}$ remains fairly constant over the experimental range of $[\mathrm{B}]_{\mathrm{o}}$ at a given temperature, as shown in Fig. 2, strongly implies that monomer-dimer self-association is dominant over oligomerization. This is plausible, due to the fact that $K=\frac{\left[\mathrm{B}_{2}\right]}{[\mathrm{B}]^{2}}=\frac{A_{\mathrm{d}} / \varepsilon_{\mathrm{d}}}{A_{\mathrm{m}}^{2} / \varepsilon_{\mathrm{m}}^{2}}$, or $\frac{A_{d}}{A_{m}^{2}}=\frac{K \varepsilon_{\mathrm{d}}}{\varepsilon_{\mathrm{m}}^{2}}$, is constant for a given 

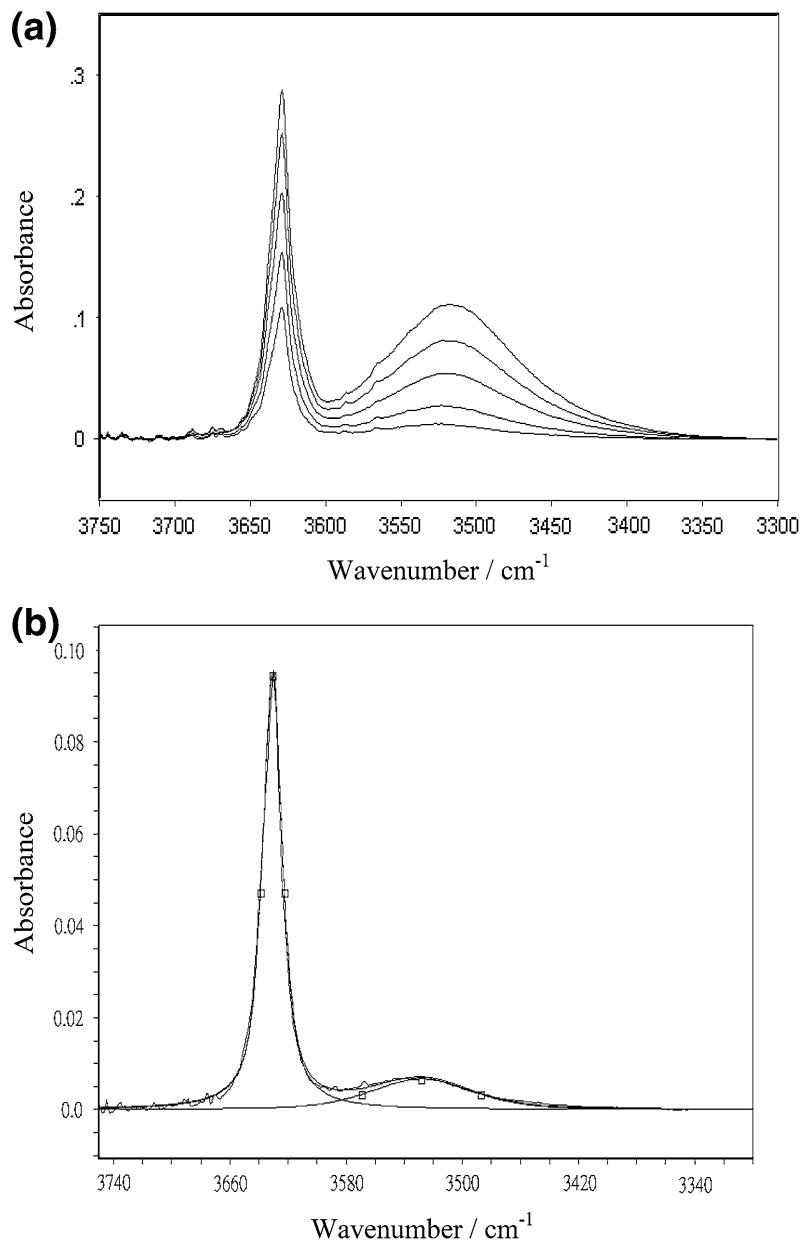

Fig. 1 a Collection of the spectra of the fundamental $\mathrm{OH}$ stretching bands for different concentrations of 2,2-dimethyl-3-ethyl-3-pentanol in $n$-octane at $298 \mathrm{~K}$ : from bottom to top: $0.2108,0.3519,0.4906,0.6368$, and $0.7692 \mathrm{~mol} \cdot \mathrm{L}^{-1}$, b curve fit for $0.2798 \mathrm{~mol} \cdot \mathrm{L}^{-1} 2,2$-dimethyl-3-ethyl-3-pentanol in $n$-octane at $298 \mathrm{~K}$

temperature since the parameters on the right hand side are only functions of temperature. Once $A_{\mathrm{m}}$ and $A_{\mathrm{d}}$ are available for different concentrations at a given temperature, we are in a position to calculate $\varepsilon_{\mathrm{m}}$ and $K$ at this temperature from the plot based on Eq. 9, and $\varepsilon_{\mathrm{d}}$ and $K$ at this temperature from the plot based on Eq. 13.

Shown in Fig. 3 are such plots for the monomer bands based on Eq. 9 and in Fig. 4 for the dimer bands based on Eq. 13 at the four temperatures. The values of $\varepsilon_{\mathrm{m}}, \varepsilon_{\mathrm{d}}$, and $K$ determined from the slope and intercept of the regressed lines at various temperatures are listed in Table 2. The values of $K$ at different temperatures can be used to obtain the standard enthalpy $\left(\Delta H^{\circ}\right)$ and entropy $\left(\Delta S^{\circ}\right)$ of dimerization through a van't Hoff plot. For this system, this is done either using $K$ from $A_{\mathrm{m}}$ or from $A_{\mathrm{d}}$. Both plots are juxtaposed in Fig. 5 for better visual comparison. The errors associated with $K, \varepsilon_{\mathrm{m}}$ and $\varepsilon_{\mathrm{d}}$ listed in Table 2, were calculated based on a standard method [32] with the assumption of equal 


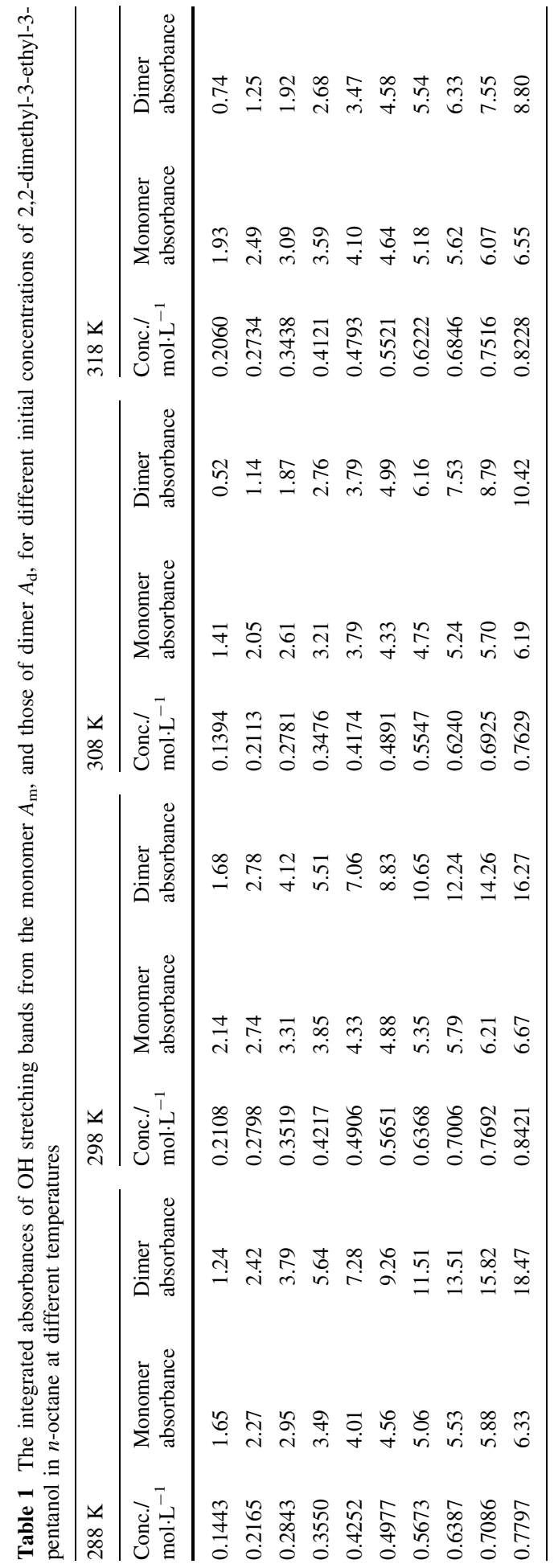




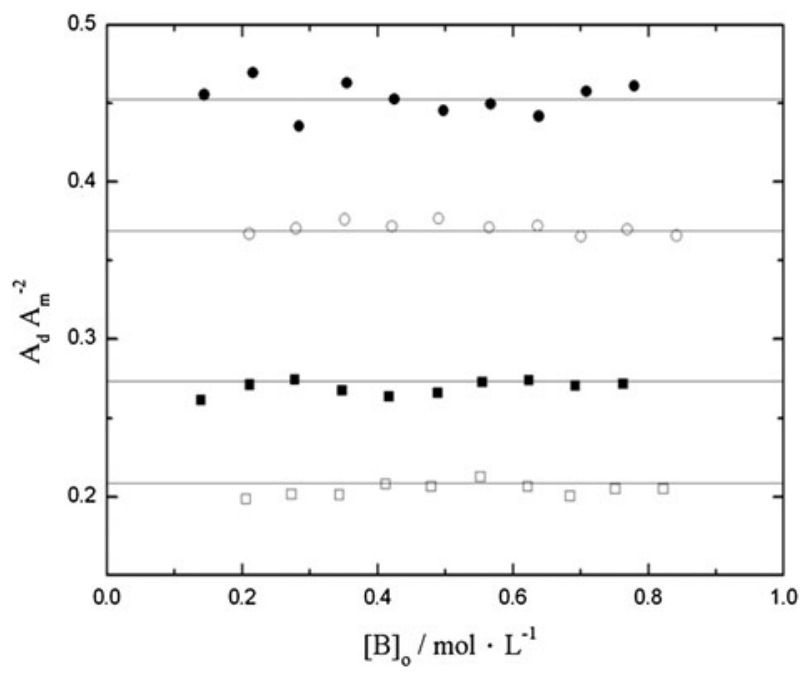

Fig. 2 Plot for comparing the theoretical lines calculated from $\frac{A_{\mathrm{d}}}{A_{\mathrm{m}}^{2}}=\frac{\varepsilon_{\mathrm{d}} b\left[\mathrm{~B}_{2}\right]}{\varepsilon_{\mathrm{m}}^{2} b^{2}[\mathbf{B}]^{2}}=\left(\frac{\varepsilon_{\mathrm{d}}}{\varepsilon_{\mathrm{m}}^{2} b}\right) K$ versus $[\mathrm{B}]_{\mathrm{o}}$, with the corresponding experimental data of $\frac{A_{\mathrm{d}}}{A_{\mathrm{m}}^{2}}$ versus $[\mathrm{B}]_{\mathrm{o}}$ of 2,2-dimethyl-3-ethyl-3-pentanol in $n$-octane at different temperatures: (filled circle) $288 \mathrm{~K}$, (open circle) $298 \mathrm{~K}$, (filled square) $308 \mathrm{~K}$, and (open square) $318 \mathrm{~K}$

variances for $A_{\mathrm{m}}$ and $A_{\mathrm{d}}$. The errors associated with $\Delta H^{\circ}$ and $\Delta S^{\circ}$ were also calculated based on a standard method [32] with unequal variances of $K$ as listed in Table 2 at each temperature. The final value of $K$ at each temperature was taken as the geometric mean of the $K$ values from Eq. 9 and from Eq. 13. The results are $0.640 \pm 0.033,0.428 \pm 0.010$, $0.283 \pm 0.008$, and $0.194 \pm 0.013 \mathrm{~L} \cdot \mathrm{mol}^{-1}$, respectively, at $288,298,308$, and $318 \mathrm{~K}$.

In order to assess the goodness of the determination we also plotted, in Fig. 6a, the data points of $y=A_{\mathrm{m}}$ versus $x=[\mathrm{B}]_{\mathrm{o}}$, juxtaposed with the theoretical curves calculated from Eq. 7, by inserting the determined values of $K$ and $\varepsilon_{m}$ for each temperature. Similar plots for $y=A_{\mathrm{d}}$ versus $x=[\mathrm{B}]_{\mathrm{o}}$ and the corresponding theoretical curves calculated from Eq. 10 are presented in Fig. 6 b.

As compared to the spectra taken by Huelsekopf and Ludwig [20] for the pure liquid at various temperatures, our monomer bands are basically in agreement with theirs in peak position, except that the free $\mathrm{OH}$ stretching of the hydrogen-bond associated complex disappears in our dilute solution case. It is a reasonable inference that the dimers in our case are mostly of the cyclic form. The bands at lower wavenumber are in disagreement, both in shape and position, due to the fact that in pure liquid state oligomerization may prevail.

It is expected that the values of $K$ determined, either from $A_{\mathrm{m}}$ based on Eq. 9 or from $A_{\mathrm{d}}$ based on Eq. 13, should be equal, since the same physical entity is referred to. As a consequence the values of $\Delta H^{\circ}$ or $\Delta S^{\circ}$ that are determined either from $A_{\mathrm{m}}$ or from $A_{\mathrm{d}}$ are also expected to be equal. This fact suggests that the discrepancy between the values of $K$ determined from $A_{\mathrm{m}}$ and from $A_{\mathrm{d}}$ at the same temperature, and the respective $\Delta H^{\circ}$ or $\Delta S^{\circ}$ from $\mathrm{A}_{\mathrm{m}}$ and $A_{\mathrm{d}}$, can serve as a consistency check.

It is obvious from Fig. 1 that the monomer bands are much sharper than the dimer bands within the experimental concentration range. Therefore, it is to be expected that the data determined from the monomer bands will be more precise than those from dimer bands. 


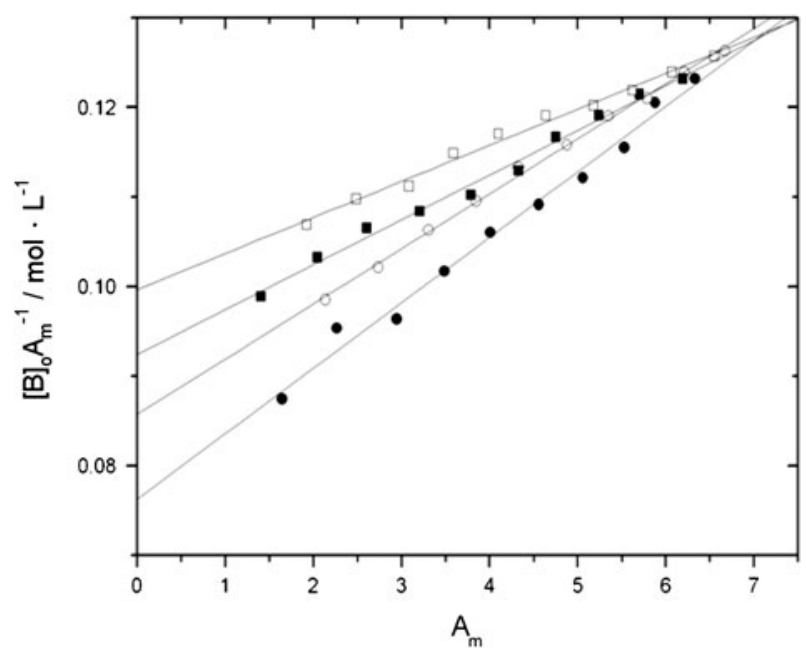

Fig. 3 Linear plot based on Eq. 9 to treat the data of monomer bands for different concentrations of 2,2dimethyl-3-ethyl-3-pentanol in $n$-octane at different temperatures: (filled circle) $288 \mathrm{~K}$, (open circle) $298 \mathrm{~K}$, (filled square) $308 \mathrm{~K}$, and (open square) $318 \mathrm{~K}$

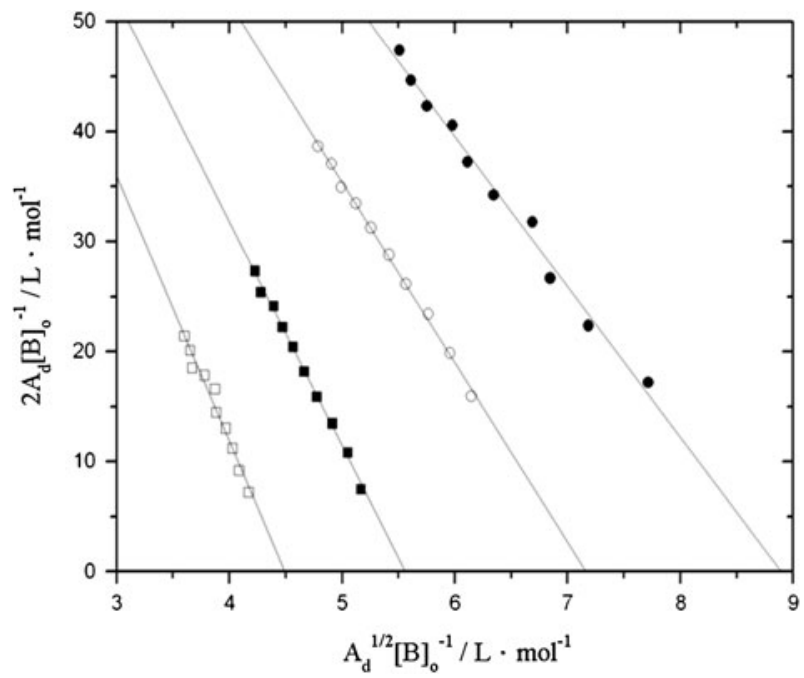

Fig. 4 Linear plot based on Eq. 13 to treat the data of dimer bands for different concentrations of 2,2dimethyl-3-ethyl-3-pentanol in n-octane at different temperatures: (filled circle) $288 \mathrm{~K}$, (open circle) $298 \mathrm{~K}$, (filled square) $308 \mathrm{~K}$, and (open square) $318 \mathrm{~K}$

However, this discrepancy should become smaller as the solute concentration increases, since the dimer bands become sharper at higher solute concentration. On account of this, it may be inappropriate to treat equilibrium constants from monomer band and from dimer band on equal footing. Nevertheless, at least the data from dimer bands can serve as a supplement to those determined from monomer bands. 
Table 2 Molar monomer absorptivities $\left(\varepsilon_{\mathrm{m}}\right)$, molar dimer absorptivities $\left(\varepsilon_{\mathrm{d}}\right)$, and dimerization constants $(K)$ for 2,2-dimethyl-3-ethyl-3-pentanol in $n$-octane at different temperatures, and the standard enthalpy $\left(\Delta H^{\circ}\right)$ and entropy $\left(\Delta S^{\circ}\right)$ of dimerization obtained from the monomer and dimer bands

\begin{tabular}{llllll}
\hline Temp/K & \multicolumn{2}{l}{ From monomer bands } & & \multicolumn{2}{l}{ From dimer bands } \\
\cline { 2 - 3 } \cline { 5 - 6 } & $K / \mathrm{L} \cdot \mathrm{mol}^{-1}$ & $\varepsilon_{\mathrm{m}} / \mathrm{L} \cdot \mathrm{cm}^{-1} \cdot \mathrm{mol}^{-1}$ & & $K / \mathrm{L} \cdot \mathrm{mol}^{-1}$ & $\varepsilon_{\mathrm{d}} / \mathrm{L} \cdot \mathrm{cm}^{-1} \cdot \mathrm{mol}^{-1}$ \\
\hline 288 & $0.629 \pm 0.028$ & $1311 \pm 21$ & & $0.651 \pm 0.039$ & $12152 \pm 327$ \\
298 & $0.418 \pm 0.008$ & $1166 \pm 7$ & & $0.438 \pm 0.012$ & $11715 \pm 156$ \\
308 & $0.294 \pm 0.010$ & $1082 \pm 8$ & & $0.273 \pm 0.009$ & $11319 \pm 190$ \\
318 & $0.202 \pm 0.007$ & $1004 \pm 6$ & & $0.186 \pm 0.019$ & $10851 \pm 562$ \\
$\Delta H^{\circ} / \mathrm{kJ}^{\prime} \mathrm{mol}^{-1}$ & $-28.61 \pm 3.88$ & & $-31.81 \pm 3.88$ & \\
$\Delta S^{\circ} / \mathrm{J} \cdot \mathrm{mol}^{-1} \cdot \mathrm{K}^{-1}$ & $-103.2 \pm 13.0$ & & & $-113.9 \pm 13.1$ & \\
\hline
\end{tabular}

Fig. 5 van't Hoff plot to obtain $\Delta H^{\circ}$ and $\Delta S^{\circ}$ for the dimerization of 2,2-dimethyl-3-ethyl-3pentanol in $n$-octane from the dimerization constants obtained at different temperatures from the monomer band (filled circle), and from the dimer band (open circle)

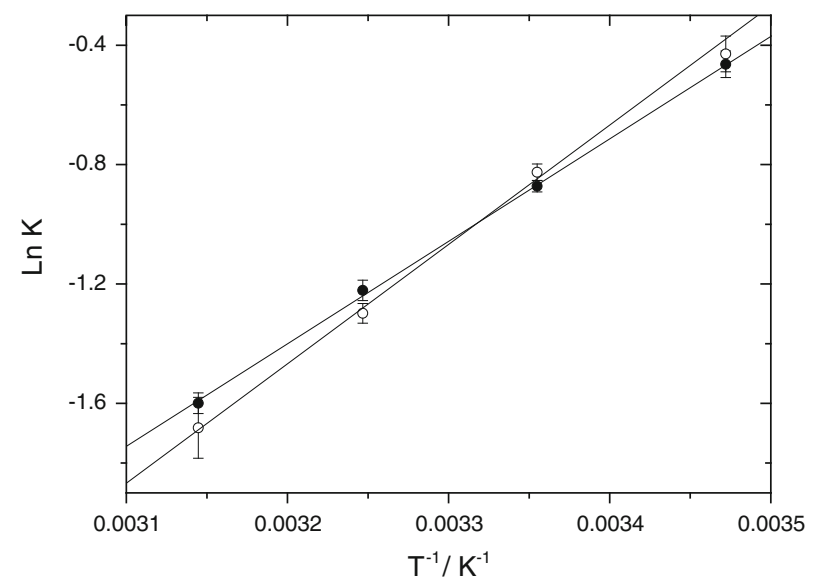

The $2 \%$ impurities in the solute may contain alkyl phenols, straight-chained and branched alcohols, which were introduced during the synthetic process. These sorts of alcohol or phenol impurities will contribute to the area of both monomer and dimer bands through the $\mathrm{OH}$ stretching of free monomers or hydrogen-bonded associates. This contribution will accordingly induce some errors in our determination.

To the best of our knowledge, most IR investigations of monomer-dimer self-association employed only the monomer bands to determine $\varepsilon_{\mathrm{m}}$ and $K$. For example, in the study of the self-association of methanol, ethanol, and tert-butanol in $\mathrm{CCl}_{4}$ solution, Liddle and Becker [33] obtained $K$ from the limiting slope of a plot of apparent absorptivity, $A_{\mathrm{m}} /[\mathrm{B}]_{\mathrm{o}}$, against $[\mathrm{B}]_{\mathrm{o}}$, through the equation:

$$
\lim _{[\mathrm{B}]_{0} \rightarrow 0}\left(\frac{d\left(\frac{A_{\mathrm{m}}}{[\mathrm{B}]_{\mathrm{o}}}\right)}{d[\mathrm{~B}]_{\mathrm{o}}}\right)=-2 K \varepsilon_{\mathrm{m}}
$$

with $\varepsilon_{\mathrm{m}}$ obtained from another limiting slope:

$$
\lim _{[\mathrm{B}]_{0} \rightarrow 0} \frac{d A_{\mathrm{m}}}{d[\mathrm{~B}]_{\mathrm{o}}}=\varepsilon_{\mathrm{m}}
$$




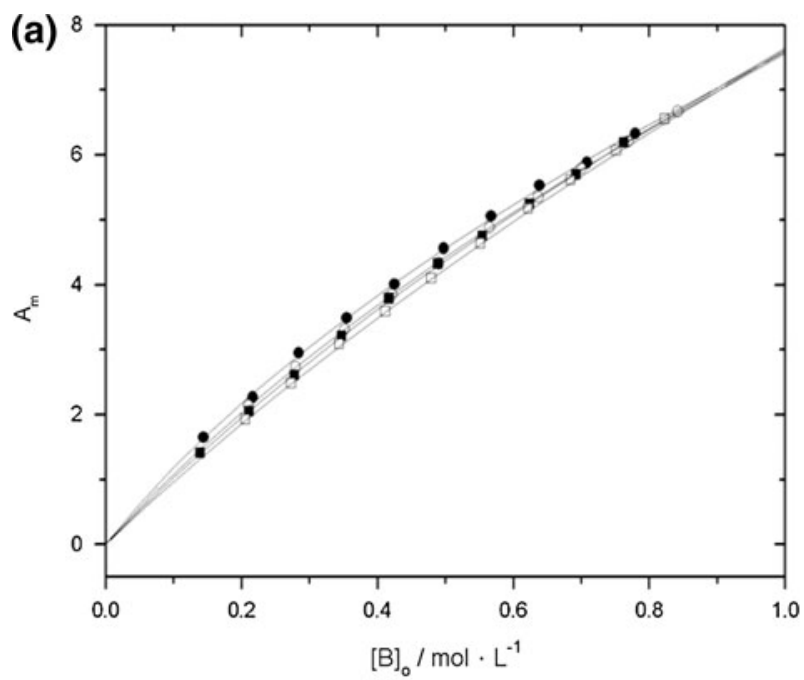

(b)

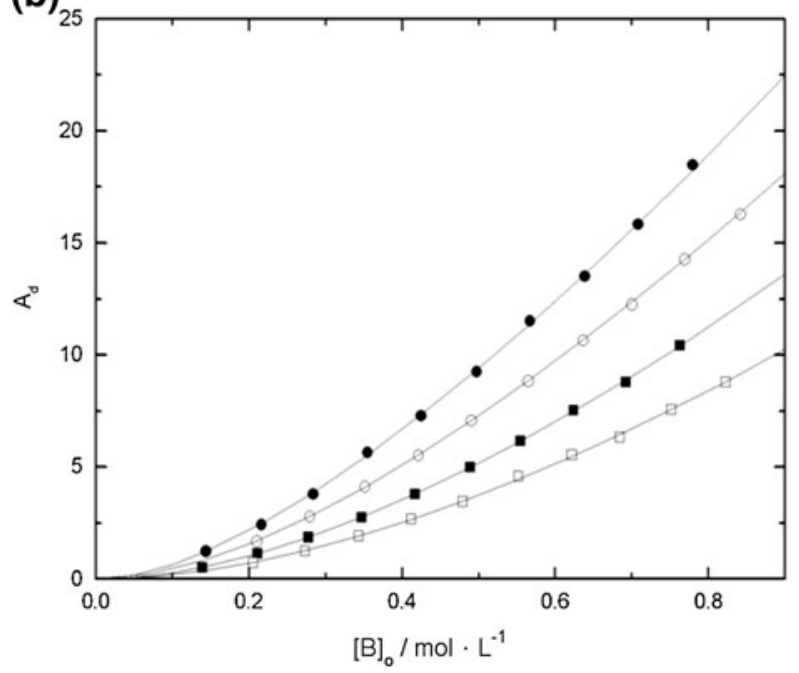

Fig. 6 Plot for comparison of the experimental data with theoretical curves: a $A_{\mathrm{m}}$ versus $[\mathrm{B}]_{\mathrm{o}}$ with the curves calculated based on Eq. 9, b $A_{\mathrm{d}}$ versus $[\mathrm{B}]_{\mathrm{o}}$ with the curves calculated based on Eq. 13, at various temperatures: (filled circle) $288 \mathrm{~K}$, (open circle) $298 \mathrm{~K}$, (filled square) $308 \mathrm{~K}$, and (open square) $318 \mathrm{~K}$

A similar method had also been adopted by Gutowsky and coworker [34] in an NMR study of the self-association of hindered phenols. The disadvantage of this method lies in the fact that a small error of the slope could induce a considerable amount of error in the determination of $\varepsilon_{\mathrm{m}}$ and $K$ if the slope is steep. Prokopenko et al. [35], in an IR study of the effect of the ring size on the self-association of lactams, fitted the data of $y=A_{\mathrm{m}}$ versus $x=[\mathrm{B}]_{\mathrm{o}}$ to the equation:

$$
A_{\mathrm{m}}=\varepsilon_{\mathrm{m}} b \frac{\sqrt{1+8 K[\mathrm{~B}]_{\mathrm{o}}}-1}{4 K}
$$


which is derivable from Eq. 7, to obtain $K$ and $\varepsilon_{\mathrm{m}}$ with the help of the LevenbergMarquardt nonlinear method. In a study of the self-association of lactams using the IR overtone, Luck [36] fitted the data of $y=A_{\mathrm{m}} /[\mathrm{B}]_{\mathrm{o}}$ versus $x=A_{\mathrm{m}}^{2} /[\mathrm{B}]_{\mathrm{o}}$ to a linear equation, which in our notation appears in the form:

$$
\frac{A_{\mathrm{m}}}{[\mathrm{B}]_{\mathrm{o}}}=\varepsilon_{\mathrm{m}}-\frac{2 K}{\varepsilon_{\mathrm{m}}}\left(\frac{A_{\mathrm{m}}^{2}}{[\mathrm{~B}]_{\mathrm{o}}}\right)
$$

This equation appears as Eq. 9 in page 163 of the above reference, it is also derivable from Eq. 8, and is used to obtain $\varepsilon_{\mathrm{m}}$ and $K$ from the slope and intercept of the regressed line. It is remarkable that all three above-mentioned methods leave the dimer band unused. Without treating the data of the dimer bands, it is impossible to obtain the molar absorptivity of the dimer. On the contrary, in addition to the data treatment of $A_{\mathrm{m}}$, our new approach also treats the data of $A_{\mathrm{d}}$ based on Eq. 13, allowing us to obtain both $\varepsilon_{\mathrm{d}}$ and $K$. Since the same $K$ can be obtained from two independent, different sources $A_{\mathrm{m}}$ and $A_{\mathrm{d}}$, the differences between these two determinations offers an additional opportunity to examine the consistency of the determination.

\section{Conclusions}

Self-association of 2,2-dimethyl-3-ethyl-3-pentanol in $n$-octane was used as a model system to illustrate a new approach to treat the data from both monomer and dimer bands. The solute compound was chosen in view of the fact that the hindrance from the neighboring bulky side chains restricts the solute molecular association to dimerization. This restriction was further examined by checking the constancy of $A_{\mathrm{d}} / A_{\mathrm{m}}^{2}$ over [B] $]_{\mathrm{o}}$ before processing the data treatment. Two new equations, Eqs. 9 and 13, have been derived to treat the integrated absorbance of the monomer bands and those of dimer bands, $A_{\mathrm{m}}$ and $A_{\mathrm{d}}$, respectively. The data treatment of $A_{\mathrm{m}}$ based on Eq. 9 allows us to obtain the molar absorptivity of the monomer bands, $\varepsilon_{\mathrm{m}}$, and the dimerization constant, $K$, at a given temperature. The data treatment of the dimer bands allows us to obtain the molar absorptivity of the dimer bands, $\varepsilon_{\mathrm{d}}$, and the dimerization constant, $K$, at a given temperature. Since the same dimerization constant at a given temperature can be obtained from two independent data sources, $A_{\mathrm{m}}$ and $A_{\mathrm{d}}$, the disparity between two determinations of $K$ provides a vehicle to assess the precision of the determination.

Acknowledgments We would like to take this opportunity to express our gratitude to the National Science Council, Taiwan, for the financial support.

\section{References}

1. Pauling, L., Corey, R.B.: Configurations of polypeptide chains with favored orientations around single bonds. Proc. Natl. Acad. Sci. USA 37, 729-740 (1951)

2. Pauling, L., Corey, R.B., Branson, H.R.: The structure of proteins: two hydrogen-bonded helical configurations of the polypeptide chain. Proc. Natl. Acad. Sci. USA 37, 205-211 (1951)

3. Waston, J.D., Crick, F.H.C.: Molecular structure of nucleic acids. Nature 171, 737-738 (1953)

4. Prausnitz, J.M., Lichtenthaler, R.N., de Azeredo, E.G.: Molecular Thermodynamics of Fluid-Phase Equilibra, 3rd edn. Prentice-Hall, New Jersey (1999)

5. Pimental, G.C., McClellan, A.L.: The Hydrogen Bond. W. H. Freeman and Company, San Francisco (1960)

6. Schuster, P., Zundel, G., Sandorfy, C. (eds.): The Hydrogen Bond, vol. 1-3. North-Holland, Amsterdam (1976) 
7. Desiraju, G.R., Steiner, T.: The Weak Hydrogen Bond. Oxford, Oxford (1999)

8. Hadži, T., Thompson, H.W.: Hydrogen Bonding. Pergamon, New York (1959)

9. Jeffery, G.A.: An Introduction to Hydrogen Bonding. New York, Oxford (1997)

10. Joesten, M.D., Schaad, L.J.: Hydrogen Bonding. Marcel Dekker, New York (1974)

11. Huyskens, P.L., Luck, W.A.P., Zeeger-Huyskens, T. (eds.): Intermolecular Forces. Springer-Verlag, Berlin (1991)

12. Errera, J., Mollet, P.: Intermolecular forces and O-H absorption bands in alcohols at $3 \mu$. Nature 138, 882 (1936)

13. Coburn Jr, W.C., Grunwald, E.: Infrared measurements of the association of ethanol in carbon tetrachloride. J. Am. Chem. Soc. 80, 1318-1322 (1958)

14. Singh, S., Rao, C.N.R.: Spectroscopic studies of self-association due to hydrogen bonding. J. Phys. Chem. 71, 1074-1078 (1967)

15. Shekaari, H., Modarress, H., Hadipour, N.: Thermodynamic investigation on self-association of alcohols in carbon tetrachloride by FT-NMR spectroscopy. J. Phys. Chem. A 107, 1891-1895 (2003)

16. Asprion, N., Hasse, H., Maurer, G.: FT-IR spectroscopic investigations of hydrogen bonding in alcoholhydrocarbon solutions. Fluid Phase Equilib. 186, 1-25 (2001)

17. FØrland, G.M., Liang, Y., Kvalheim, O.M., Hoiland, H., Chazy, A.: Associative behavior of benzyl alcohol in carbon tetrachloride solutions. J. Phys. Chem. B 101, 6960-6969 (1997)

18. FØrland, G.M., Libnau, F.O., Kvalheim, O.M., HØiland, H.: Self-association of medium-chain alcohols in $n$-decane solutions. Appl. Spectrosc. 50, 1264-1272 (1996)

19. Nodland, E.: Studies of self-association in alcohols (ROH) as functions of concentrations and temperature. Part II: curve resolution of concentration series. Appl. Spectrosc. 54, 1339-1349 (2000)

20. Huelsekopf, M., Ludwig, R.: Hydrogen bonding in a sterically hindered alcohol. J. Mol. Liq. 98, 163-171 (2002)

21. Laenen, R., Simeonidis, K.: Vibrational energy migration in a hydrogen-bonded dimer probed with ultrafast time-resolved spectroscopy. Chem. Phys. Lett. 292, 631-637 (1998)

22. Brink, G., Campbell, C., Glasser, L.: Dielectric studies of molecular association. Concentration dependence of the dipole moment of 2,3,4-trimethyl-3-pentanol in solution. J. Phys. Chem. 80, 2560-2563 (1976)

23. Laenen, R., Simeonidis, K., Ludwig, R.: Investigation of an H-bonded dimer: calculations of bonding structures and temperature dependence of the librational substructure of the OH-stretching band. J. Chem. Phys. 111, 5897-5904 (1999)

24. Laenen, R., Simeonidis, K.: Towards a more detailed understanding of the OH-stretching bands of H-bonded liquids. Chem. Phys. Lett. 290, 94-98 (1998)

25. Johari, G.P., Dannhauser, W.: Dielectric study of intermolecular association in sterically hindered octanol isomers. J. Phys. Chem. 72, 3273-3276 (1968)

26. Luo, W.C., Lay, J.L., Chen, J.S.: NMR study of hydrogen bonding association of some sterically hindered alcohols in carbon tetrachloride, chloroform and cyclohexane. Z. Phys. Chem. 216, 829-843 (2002)

27. Chen, J.S., Shirts, R.B.: Iterative determination of the NMR monomer shift and dimerization constant in a self-associating system. J. Phys. Chem. 89, 1643-1646 (1985)

28. Chen, J.S., Rosenberger, F.: Accurate NMR data evaluation for monomer shift, dimer shift and dimerization constant in a self-associating system. Tetrahedron Lett. 31, 3975-3978 (1990)

29. Chen, J.S.: NMR study of $\hat{I} \mu$-caprolactam in various solvents. Graphical determination of monomer shift, dimer shift and dimerization constant from the dilution shift data. J. Chem. Soc. Faraday Trans. 90, 717-720 (1994)

30. Mark, H.F., Othmer, D.F., Overberger, C.G., Seaborg, G.T.: In: Kirk-Othmer, (ed.) Encyclopedia of chemical technology, vol. 12, 4th edn, p. 325. Wiley, New York (1999)

31. Budavari, S. (ed.): Merck Index, 11th edn, p. 6674. Merck and Co., Inc., New Jersey (1989)

32. Bevington, P.R., Robinson, D.K.: Data Reduction and Error Analysis, 3rd edn. McGraw-Hill, Boston (2003)

33. Liddel, U., Becker, E.D.: Infra-red spectroscopic studies of hydrogen bonding in methanol, ethanol, and $t$-butanol. Spectrochim. Acta 10, 70-84 (1957)

34. Somers, B.G., Gutowsky, H.S.: Nuclear magnetic resonance studies of hydrogen bonding in hindered phenols. J. Am. Chem. Soc. 85, 3065-3072 (1963)

35. Prokopenko, N.A., Bethea, I.A., Clemens, C.J., Klimek, A., Wargo, K., Spivey, C., Waziri, K., Grushow, A.: The effect of structure on hydrogen bonding: hydrogen bonded lactam dimers in $\mathrm{CCl}_{4}$. Phys. Chem. Chem. Phys. 4, 490-495 (2002)

36. Luck, W.P.: IR-overtone vibration spectroscopy, chap. 7. In: Huyskens, P.L., Luck, W.A.P., ZeegerHuyskens, T. (eds.) Intermolecular Forces. Springer-Verlag, Berlin (1991) 